\title{
Optimal Control with Linear Integer Programming for Reducing the Energy Consumption of Interdependent Mixing Machines in Foundry
}

\author{
$1^{\text {st }}$ Alexander Rose \\ IKME - Advanced Control \\ Hannover University of \\ Applied Science and Arts \\ Hannover, Germany \\ alexander.rose@hs-hannover.de
}

\author{
$2^{\text {nd }}$ Axel Schild, \\ $3^{\text {rd }}$ Bennet Luck \\ Automation Solutions Energy Sector \\ IAV GmbH \\ Gifhorn, Germany \\ \{axel.schild, bennet.luck\}@iav.de
}

\author{
$4^{\text {th }}$ Martin Grotjahn \\ IKME - Advanced Control \\ Hannover University of \\ Applied Science and Arts \\ Hannover, Germany \\ martin.grotjahn@hs-hannover.de
}

\begin{abstract}
This paper presents a novel approach for modelling the energy consumption of the coupled parallel moulding sand mixers of a foundry as an optimal control problem. The minimization of energy consumption is optimized by scheduling the mixing processes in a linear integer programming scheme. The sand flow through the foundry's sand preparation is characterized by a physical model. This model considers the sand demand of the moulding machine as disturbance, the stored sand masses in the mixer hoppers and machine hoppers, respectively. The novel approach of handling dwell-times for dosing, mixing and transport processes using dead-time systems and constraint pushing allows the application of a linear model. The formulation of the optimal control problem aims at real-time application as model predictive control at the production plant. Initial application results indicate an improvement in energy consumption of approximately $8 \%$.
\end{abstract}

Index Terms-model predictive control, linear integer programming, scheduling, dwell-time, constraint pushing, soft constraint, batch-wise parallel process, foundry

\section{INTRODUCTION}

Electrical energy consumption in a foundry is an important factor to reduce costs and tackle the rising requirements of environmental legislation. The sand preparation is an essential driver for energy consumption especially in green sand casting with cupola furnace. The moulding sand is strongly affected by environmental conditions [1]. The mixed sand is stored in the hoppers. Storing the sand needs space in the production plant. Therefore, the dimension of the sand hoppers after the mixers is small. Thus, the mixers have to react quickly to the changes in moulding sand demand. In practice, the operation strategy is typically controlled by a few discrete measurements of sand level in the hoppers.

In the exemplary foundry Heinrich Meier Eisengießerei GmbH \& Co. KG in Rahden, both sand mixers operate batchwise. The operation strategy aims at maximum filling of the hoppers without taking energy cost into account. Due to process stability the mixing time is fix [2]. The operating strategy generates idle times, while the mixers are running

\footnotetext{
The European Regional Development Fund co-financed this research.
}

and waiting for the next batch. In international comparison, energy costs and capacity utilization in Germany are high [3]. In the industry with energy-intensive processes, the efficient use of energy will be a decisive competitive advantage [4], [5]. Actual exemplary investigations in the sand preparation of a foundry show a potential for energy saving up to $10 \%$ [6]. Investigations on the energy consumption of the mixing process at the exemplary foundry confirm this potential. Therefore, managing the sand flow through the sand preparation and switching-off the mixer during idle time offers optimization potential.

Scheduling for production planning in foundries with binary integer programming for optimizing the production planing can be found in [7]. In this approach the trade off between model complexity and calculation time is discussed. An overview of various optimization approaches and an improved variable neighbourhood search to find a near-optimal solution for batch delivery planning in steel production can be found in [8]. A tailored branch and bound algorithm to reduce the computational effort when scheduling parallel processes in a steel foundry is presented in [9]. Scheduling of parallel industrial processes with mixed integer programming are shown in [10], [11]. The related work aims at offline production planing. A stochastic approach to energy efficient switching of a machine tool using buffer level can be found in [12] and related work.

In this paper, we discuss a novel optimal control approach for real-time application on the mixing process at the production plant. The novel approach for integrated modelling of the processes in sand preparation, from dosing in the scale to moulding in the machine, allows the optimization of the mixers' energy consumption. The optimization model captures the dependence of batch processes, sand flow and buffered sand masses on the energy consumption. The essential challenge is handling of dwell-times for sand dosing, mixing process and sand transport. We tackle this challenge by using discrete dead-time systems. Initial application results at the real production plant indicate an improvement in energy consumption of approximately $8 \%$. 
Section II introduces the mathematical representation of the optimal control model with a linear model approach. In section III the optimal control problem with the economic formulation of the cost function including the energy demand of the moulding sand mixers and the constraint treatment is presented. The results of the optimal control and first test results at the production plant, presented in section IV, suggest an advantage in energy consumption.

\section{Optimal Control Model}

First the process is discussed and the optimal control model with state variables, manipulated variables and the process disturbance is presented. The real process at the production plant includes dosing the sand into a scale, mixing in the mixers to adjust the moulding properties, transporting the sand to the machine hopper and buffering the sand for the moulding process, see Fig. 1. We use a time-discrete model formulation with time-steps $k$. For solving the optimal control problem we consider a simple process model, which

- represents the scale as two interdependent scales,

- captures the aspects of dosing, mixing and sand transport as discrete dead-time system,

- omits the influence of the mixer hopper extraction belts

- and combines the two machine hoppers into one.

Starting point of the sand flow is the scale, see Fig. 1 (top). In the real process, one scale doses the sand batch-wise for the two mixers. The scale can only fill one mixer simultaneously.

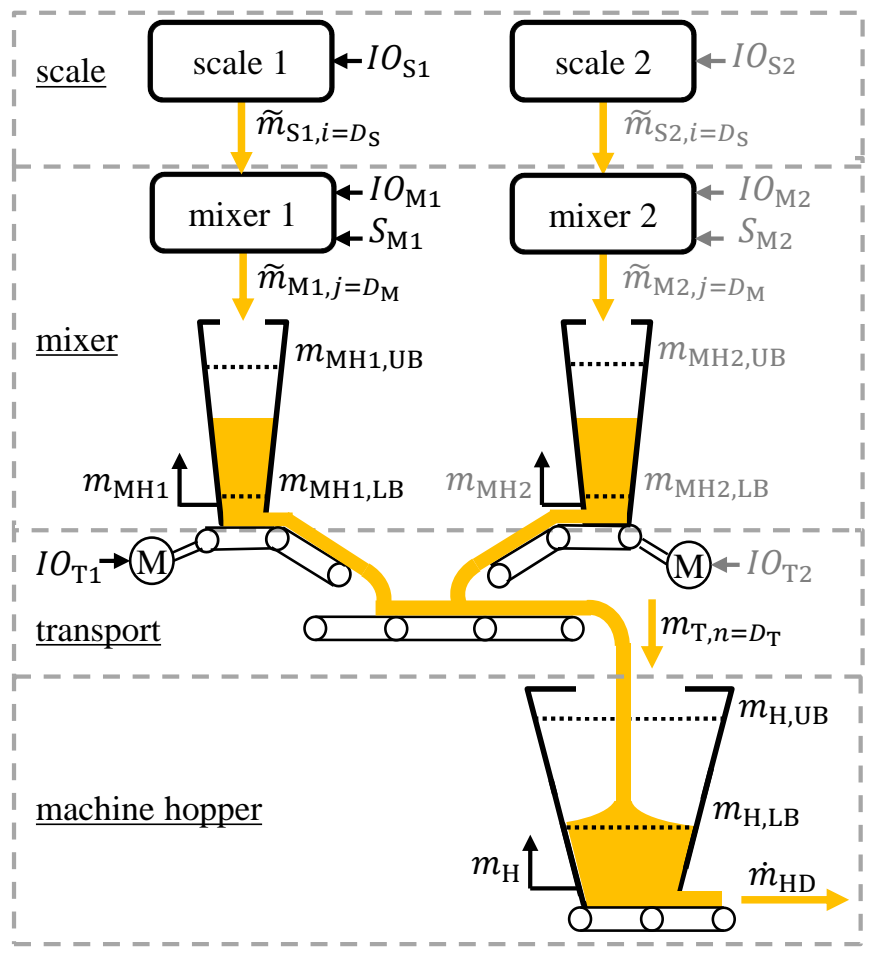

Fig. 1. Schematic process description with the scales (top), the mixers, mixer hoppers and sand transport belt (middle) and the machine hoppers combined into one (bottom)
TABLE I

STATES AND INPUTS

\begin{tabular}{|c|c|c|}
\hline & Description & Units \\
\hline$k$ & discrete time-steps & \\
\hline $\bar{K}$ & prediction horizon & \\
\hline 0 & $\begin{array}{l}\text { abbreviation for scales and mixers }\{1,2\} \\
c \text { : counting variable for scales and mixers }\end{array}$ & \\
\hline \multicolumn{3}{|c|}{ 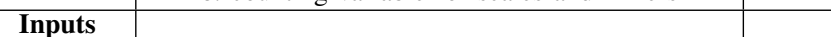 } \\
\hline$I O_{\mathrm{So}}$ & start dosing scales $\{0,1\}$ & - \\
\hline$I O_{\mathrm{Mo}}$ & switch mixers states $\{-1,0,1\}$ & - \\
\hline$I O_{\mathrm{To}}$ & ON/OFF extraction belts $\{0,1\}$ & - \\
\hline \multicolumn{3}{|l|}{ States } \\
\hline$\tilde{m}_{\mathrm{So}, i}$ & $\begin{array}{c}D_{\mathrm{S}} \text { normalized masses dosing each mass }\{0,1\} \\
i \text { : counting variable for the dosing process }\end{array}$ & - \\
\hline$S_{\mathrm{Mo}}$ & mixers' states $\{0,1\}$ & - \\
\hline$\tilde{m}_{\mathrm{M}, j}$ & $\begin{array}{c}D_{\mathrm{M}} \text { normalized masses mixing each mass }\{0,1\} \\
j \text { : counting variable for the mixing process }\end{array}$ & - \\
\hline$m_{\mathrm{MH} \circ}$ & masses in the mixer hoppers & $\mathrm{kg}$ \\
\hline$m_{\mathrm{T}, n}$ & $\begin{array}{c}\text { mass on transport; number states: } D_{\mathrm{T}} \\
n \text { : counting variable for the transport process }\end{array}$ & $\mathrm{kg}$ \\
\hline$m_{\mathrm{H}}$ & mass in the machine hopper & $\mathrm{kg}$ \\
\hline$\dot{m}_{\mathrm{HD}}$ & mass flow to the moulding machine (disturbance) & $\mathrm{kg} / \mathrm{s}$ \\
\hline \multicolumn{3}{|c|}{ 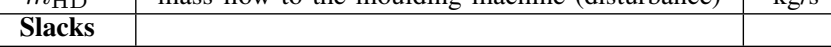 } \\
\hline$s_{\mathrm{So}}$ & buffering sand in scales each $\{0,1\}$ & - \\
\hline$s_{\mathrm{MSo}}$ & mixers' starts each $\{0,1\}$ & - \\
\hline$s_{\mathrm{MH}, \mathrm{LB}}$ & soft constraints mass mixer hoppers lower bound & - \\
\hline$s_{\mathrm{MHo}, \mathrm{UB}}$ & soft constraints mass mixer hoppers upper bound & - \\
\hline$s_{\mathrm{H}, \mathrm{LB}}$ & soft constraint mass machine hopper lower bound & - \\
\hline$s_{\mathrm{H}, \mathrm{UB}}$ & soft constraint mass machine hopper upper bound & - \\
\hline
\end{tabular}

Before dosing, the scale output switches to one of the two mixers. In the model we treat this with two interdependent scales. The starts of the dosages are controllable with the input variables $I O_{\mathrm{So}}$, see table I (section Inputs). The variables of the two scales are abbreviate with $\circ=\{1,2\}$. The dwell time in the scale due to dosing is modelled as a dead-time system with $D_{\mathrm{S}}$ discrete time-steps, see table II (section Parameters). Modelling dwell-time for dosing as dead-time system results in $i=D_{\mathrm{S}}$ state variables, see table I (section States). The process may prohibit the discharge of sand into the mixer, e.g. if the mixer cannot start processing. Therefore, a softconstrained formulation with slack variables [13] $s_{\mathrm{So}} \in\{0,1\}$ allows buffering sand in the scale, see table I section Slacks. Within the dead-time system, the entire mass of the batch is transferred with each time step from the actual state to

TABLE II

PARAMETERS AND BOUNDS

\begin{tabular}{|c|c|c|c|}
\hline & Description & Units & Size \\
\hline Parameters & dosing time scale in steps & - & \\
\hline$D_{\mathrm{S}}$ & mixing time in steps & - & 9 \\
\hline$D_{\mathrm{M}}$ & mass batch mixer & $\mathrm{kg}$ & 6000 \\
\hline$C_{\mathrm{S} \circ}$ & transport time in steps & - & 2 \\
\hline$D_{\mathrm{T}}$ & mass extraction mixer hoppers & $\mathrm{kg}$ & $58 \Delta t$ \\
\hline$C_{\mathrm{T} \circ}$ & sample time & $\mathrm{s}$ & 15 \\
\hline$\Delta t$ & & & \\
\hline Bounds & mass mixer hoppers lower bound & $\mathrm{kg}$ & 0 \\
\hline$m_{\mathrm{MH}, \mathrm{LB}}$ & mass mixer hoppers upper bound & $\mathrm{kg}$ & 14000 \\
\hline$m_{\mathrm{MH}, \mathrm{UB}}$ & mass machine hopper lower bound & $\mathrm{kg}$ & 3000 \\
\hline$m_{\mathrm{H}, \mathrm{LB}}$ & mass machine hopper upper bound & $\mathrm{kg}$ & 19000 \\
\hline$m_{\mathrm{H}, \mathrm{UB}}$ & \multicolumn{2}{|l}{} \\
\hline & & &
\end{tabular}


the following state until the dosing time is reached. Due to the process the sand mass per batch is fixed. Therefore, we normalize the state variables with the fixed sand mass per batch to $\{0,1\}$ and store the mass information in a variable $C_{\text {So }}$. The normalized masses are marked with a tilde. Through this modelling the state variables can be treated as binary in the optimization. The model for the entire dosing process with $i=D_{\mathrm{S}}$ states denotes

$$
\begin{aligned}
& \tilde{m}_{\mathrm{S} \circ, 1}(k+1)=I O_{\mathrm{S} \circ}(k) \\
& \tilde{m}_{\mathrm{S}, i}(k+1)=\tilde{m}_{\mathrm{S} \circ, i-1}(k), \quad i=2, \cdots, D_{\mathrm{S}}-1 \\
& \tilde{m}_{\mathrm{S}, D_{\mathrm{S}}}(k+1)=\tilde{m}_{\mathrm{S}, D_{\mathrm{S}-1}}(k)+s_{\mathrm{S} \circ}(k) .
\end{aligned}
$$

At the end of the dosing process the sand is emptied into the related mixer and the mixing process starts immediately. The mixers prepare the sand for the moulding machine, see Fig. 1 (middle). Each mixer has a hopper to buffer the sand after the mixing process. The state of the mixer $S_{\mathrm{M} \text { o }}$ can be zero $(O F F)$, no current or one $(O N)$, ready for process. The control variable $I O_{\mathrm{Mo}} \in\{-1,0,1\}$ switch the mixer state:

$$
S_{\mathrm{M} \circ}(k+1)=S_{\mathrm{M} \circ}(k)+I O_{\mathrm{M}}(k) .
$$

The specification for the sand quality requires emptying the mixer at the end of process time. The mixing process is also modelled by a dead-time system based on normalized masses. This results in $j=D_{\mathrm{M}}$ state variables reads

$$
\begin{aligned}
& \tilde{m}_{\mathrm{M} \circ, 1}(k+1)=\tilde{m}_{\mathrm{S} \circ, D_{\mathrm{S}}}(k)-s_{\mathrm{S} \circ}(k) \\
& \tilde{m}_{\mathrm{M} \circ, j}(k+1)=\tilde{m}_{\mathrm{M}, j-1}(k), \quad j=2, \cdots, D_{\mathrm{M}} .
\end{aligned}
$$

At the end of the mixing process, the sand mass is emptied into the related mixer hopper. From the sand mass in the hoppers $m_{\mathrm{MH}}$ infinitesimal short extraction belts with binary control $I O_{\mathrm{T} 。} \in\{0,1\}$ extract sand by the factor $C_{\mathrm{To}}$, see table II section Parameters. The sand mass in the mixer hoppers results as

$$
\begin{aligned}
m_{\mathrm{MH}}(k+1)= & m_{\mathrm{MH}}(k)+C_{\mathrm{S} \circ} \tilde{m}_{\mathrm{M}, D_{\mathrm{M}}}(k) \\
& -C_{\mathrm{T} \circ} I O_{\mathrm{T} \circ}(k) .
\end{aligned}
$$

Both belts convey sand from the hoppers to one constantly running transport belt. This results in the mass on the transport belt, modelled as dead-time system, with $n=D_{\mathrm{T}}$ states:

$$
\begin{aligned}
& m_{\mathrm{T}, 1}(k+1)=\sum_{\circ \in\{1,2\}} C_{\mathrm{T} \circ} I O_{\mathrm{T} \circ}(k) \\
& m_{\mathrm{T}, n}(k+1)=m_{\mathrm{T}, n-1}(k), \quad n=2, \cdots, D_{\mathrm{T}} .
\end{aligned}
$$

At real production plants, the sand can be divided among several machine hoppers at the end of the transport belt. Dividing the sand into these machine hoppers is an underlying control problem and typically varies with the production. Thus, in the model, the sand flows to one common machine hopper. The fluctuating sand demand of the moulding machine also influences the machine hopper mass. The sand demand is modelled as disturbing mass flow $\dot{m}_{\mathrm{HD}}$ with sample time $\Delta t$. The mass in the machine hopper can be represented by

$$
m_{\mathrm{H}}(k+1)=m_{\mathrm{H}}(k)+m_{\mathrm{T}, D_{\mathrm{T}}}(k)-\dot{m}_{\mathrm{HD}}(k) \Delta t .
$$

\section{Optimal Control Problem}

The optimal control problem contains the economic cost function and the constraint treatment. The economic formulation of the costs $J$ contains the buffering of sand in scale $h_{\mathrm{S}}$, the mixers' energy consumption (starting $h_{\mathrm{MS}}$ and idling $\left.h_{\mathrm{MI}}\right)$ and the hopper restrictions $h_{\mathrm{MH}}$ and $h_{\mathrm{H}}$, respectively. The energy demand of the sand transport is constant and is neglected in the cost function. Penalizing buffering of sand in the scale, see table III, yields

$$
h_{\mathrm{S}}=\sum_{\circ \in\{1,2\}} w_{\mathrm{S} \circ} s_{\mathrm{S} \circ}(k) .
$$

The dosing process is restricted. During the dosing time of one scale the other scale is locked by

$$
\sum_{\circ \in\{1,2\}} \sum_{i=k-D_{\mathrm{S}}}^{k} I O_{\mathrm{So}}(i) \leq 1
$$

The information for the time steps $k-D_{\mathrm{S}}<0$ is stored in the normalized masses $\tilde{m}_{\mathrm{So}, i}$. The formulation $(1 \mathrm{a}-1 \mathrm{c})$ yields the restriction $D_{\mathrm{S}} \in \mathbb{N}_{>1}$. Thus, the lock condition for the scales (8) can be reformulated as

$$
\sum_{\circ \in\{1,2\}}\left[I O_{\mathrm{S} \circ}(k)+\sum_{i=1}^{D_{\mathrm{S}}} \tilde{m}_{\mathrm{S} \circ, i}(k)\right] \leq 1 .
$$

The energy consumption of the mixers includes the cost for starting the mixers and the cost for idle times. The energy consumption for the mixing process itself is constant because of

- constant sand masses per batch,

- a invariable mixing time,

- equal processing costs per mixer

- and invariant number of batches over a infinitely long prediction horizon.

Therefore, the energy costs for the mixing process is eliminated for the cost function. Through pushing constraints $s_{\text {MSo }} \in\{0,1\}$, starting the mixers are separated from the switching variables. Due to mixing process time, sand from a batch near the end of the prediction horizon $K \in \mathbb{N}$ has no impact on the hopper masses. Therefore, the mixers shut down at the end of the prediction horizon. As a result, the optimizer tends to push switch-offs to the end. Adding terminal constraints to the cost function eliminate this effect. The

TABLE III

WEIGHTS

\begin{tabular}{|c|c|c|c|}
\hline & Description & Units & Value \\
\hline$w_{\text {S॰ }}$ & buffering sand in scales & - & $10^{6}$ \\
\hline$w_{\text {MS॰ }}$ & mixers' starts & - & 2025 \\
\hline$w_{\text {MI॰ }}$ & mixer idle times & - & $75 \Delta t$ \\
\hline $\mathbf{w}_{\mathrm{MH}}$ & soft constraints mixer hoppers & {$[--]$} & {$\left[10^{6} 10^{6}\right]$} \\
\hline $\mathbf{w}_{\mathrm{H}}$ & soft constraints machine hopper & {$[--]$} & {$\left[10^{6} 10^{6}\right]$} \\
\hline
\end{tabular}


penalty for the mixers' starting cost $w_{\mathrm{MS}}$ comprises energy consumption and wear.

$$
\begin{array}{r}
h_{\mathrm{MS}}=\sum_{\circ \in\{1,2\}} w_{\mathrm{MS}} s_{\mathrm{MS}}(k) \\
\text { s.t. } I O_{\mathrm{M} \circ}(k)-s_{\mathrm{MS}}(k) \leq 0 \\
s_{\mathrm{MS} \circ}(K)=1 .
\end{array}
$$

Until the actual batch is in process, the mixers are disabled for the next batch by

$$
\sum_{j=1}^{D_{\mathrm{M}}} \tilde{m}_{\mathrm{M}, j}(k) \leq 1 .
$$

During the mixing process, the mixers have to be switched on. While the last mass is being dosed in the scale, the mixers have to be started for the mixing process. Therefore switching off the mixers is blocked by

$$
\tilde{m}_{\mathrm{S} \circ, D_{\mathrm{S}}}(k)+\sum_{j=1}^{D_{\mathrm{M}}} \tilde{m}_{\mathrm{M}, j}(k) \leq S_{\mathrm{M}}(k) .
$$

Idle times occur when the mixer states are $O N$ and waiting for the next batch. Combining the constraints (11) and (12), the cost of mixer idle times penalized by $w_{\text {MIo leads to: }}$

$$
\begin{gathered}
h_{\mathrm{MI}}=\sum_{\circ \in\{1,2\}} w_{\mathrm{MI} \circ}\left[S_{\mathrm{M} \circ}(k)-\sum_{j=1}^{D_{\mathrm{M}}} \tilde{m}_{\mathrm{M}, j}(k)\right] \\
\text { s.t. } \sum_{j=1}^{D_{\mathrm{M}}} \tilde{m}_{\mathrm{M}, j}(k) \leq S_{\mathrm{M} \circ}(k) \\
\tilde{m}_{\mathrm{S} \circ, D_{\mathrm{S}}}(k) \leq S_{\mathrm{M} \circ}(k) .
\end{gathered}
$$

The hopper masses are optimization variables. However, in a soft-constrained formulation the violation of the hopper mass restrictions lead to step costs. The production requires emptying the hoppers at the end of the day. The optimizer tends to empty the hoppers within the horizon. With an infinite prediction horizon, here one production day, terminal cost can be omitted. For industrial model application, the optimization has to be performed in real-time. Therefore, the finite horizon has to be much shorter than a production day and a terminal constraint is added. The entire costs for the hopper masses read

$$
\begin{aligned}
& h_{\mathrm{MH}}= \sum_{\circ \in\{1,2\}} \mathbf{w}_{\mathrm{MH}}\left[\begin{array}{l}
s_{\mathrm{MH}, \mathrm{LB}}(k) \\
s_{\mathrm{MH}, \mathrm{UB}}(k)
\end{array}\right] \\
& \text { s.t. } m_{\mathrm{MH}, \mathrm{LB}}-s_{\mathrm{MH}, \mathrm{LB}}(k) \leq m_{\mathrm{MH}}(k) \\
& m_{\mathrm{MH}, \mathrm{UB}}+s_{\mathrm{MH}, \mathrm{UB}}(k) \geq m_{\mathrm{MH}}(k) \\
& s_{\mathrm{MH}, \mathrm{LB}}(k), s_{\mathrm{MH}, \mathrm{UB}}(k) \geq 0, \\
& h_{\mathrm{H}}= \mathbf{w}_{\mathrm{H}}\left[\begin{array}{c}
s_{\mathrm{H}, \mathrm{LB}}(k) \\
s_{\mathrm{H}, \mathrm{UB}}(k)
\end{array}\right] \\
& \text { s.t. } \quad m_{\mathrm{H}, \mathrm{LB}}-s_{\mathrm{H}, \mathrm{LB}}(k) \leq m_{\mathrm{H}}(k) \\
& m_{\mathrm{H}, \mathrm{UB}}+s_{\mathrm{H}, \mathrm{UB}}(k) \geq m_{\mathrm{H}}(k) \\
& s_{\mathrm{H}, \mathrm{LB}}(k) s_{\mathrm{H}, \mathrm{UB}}(k) \geq 0 \\
& m_{\mathrm{H}}(0)-m_{\mathrm{H}}(K) \leq 0 .
\end{aligned}
$$

Thus, the resulting economic cost formulation is completely linear. The entire cost function results in

$$
\begin{aligned}
& \underset{\substack{\text { Inputs, } \\
\text { Slacks } \\
\forall k \leq K}}{\min } J=h_{\mathrm{S}}+h_{\mathrm{MS}}+h_{\mathrm{MI}}+h_{\mathrm{MH}}+h_{\mathrm{H}} \\
& \text { s.t. }(7),(9),(10),(13-14 b) .
\end{aligned}
$$

\section{RESULTS}

The actual operation strategy of the mixers aims at filling the machine hoppers of the plant. Therefore, both mixers produce sand in alternating order. The extraction belts of the mixer hoppers stop, when the machine hoppers are full. The mixer hoppers are filled to their maximum and then the next batch of sand is buffered in the scale. At the beginning of dosage, the mixer for this batch is determined. The mixing process starts immediately, when the mixer related hopper can store the sand. The mixer runs in idle until the next batch starts.

The improvement of the optimal control approach ${ }^{\dagger}$ is reduction of energy consumption. Furthermore, first results of the real-time test of the optimal control problem, in a model predictive control scheme, are illustrated on the production plant. For the parametrization of the optimal control problem see table II. The parametrization of the weights is shown table III. A reduced complexity of the optimization model leads to lower calculation effort. The model complexity is proportional to the number of dead-time steps for dosing $D_{\mathrm{S}}$, mixing $D_{\mathrm{M}}$ and transport $D_{\mathrm{T}}$, which have to be integer. The minimal numbers of dead-time steps and the sample time $\Delta t$ result from the process conditions at the production plant.

\section{A. Solving the Optimal Control Problem}

The disturbance, namely the moulding sand demand of the plant, varies due to production changes and interruptions, respectively. Especially interruptions are not predicted. Therefore, the optimal control is performed with an constant moulding sand demand as disturbance. The value of the disturbance is $61 \mathrm{~kg} / \mathrm{s}$ and corresponds to an average moulding sand demand of the production plan. The prediction horizon is 160 steps (40 minutes). The sample time is fixed to 15 seconds per step. The mixer states, see Fig. 2 (top), the starts of sand dosing (second) and the inputs for the transport belts (third and fourth) contain zeros for state $O F F$ and ones for state $O N$. In the prediction horizon both mixers shut down for several consecutive time steps, see Fig. 2 (top, sectors A and A*). In this example the mixers run without idle. In the actual heuristic control the mixers are always switched on and ready for the next batch.

The starts of sand dosing are related to the mixer states. In the actual control, both mixers produce sand in alternating order. In comparison, the optimal control approach leads to consecutive doses, see Fig. 2 (second, sectors B and B*). Switching the inputs for the extraction belts control the sand transport between the mixer hoppers and the machine hopper,

\footnotetext{
${ }^{\dagger}$ Solver for the optimal control problem is CPLEX ILOG v12.10. The model is build in matlab R2020b and the interaction between them is performed with YALMIP [14].
} 
see Fig. 2 (third, forth). In the actual heuristic control the inputs would be $O N$ as long as the machine hopper mass is below the maximum. The mass in the hopper also reacts on the sand transport. The masses in both mixer hoppers $m_{\mathrm{MH}}$ almost fluctuate in the full range between minimum and maximum bound. The mass in the machine hopper $m_{\mathrm{H}}$ varies less and is in general below $50 \%$ of the maximum mass, see Fig. 2 (bottom, line C). By extending the prediction horizon the range of fluctuation is relatively constant. This effect is unexpected and could be a result of the interdependent batchwise mixing processes or the relatively small capacity of the machine hopper in relation to the mixer hoppers, respectively.
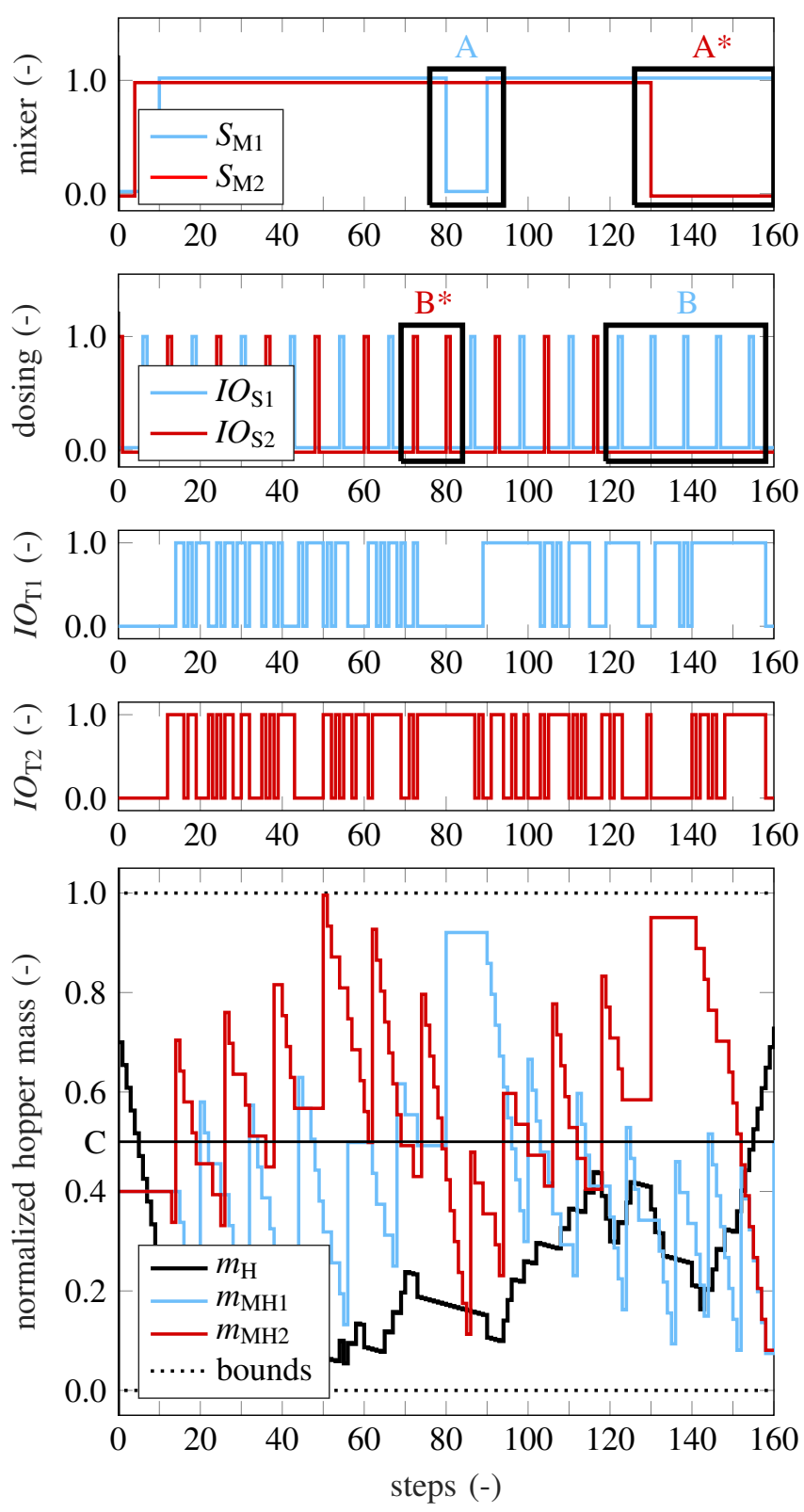

Fig. 2. Optimal control with the mixer states (top), dosing starts (second), the inputs for the transport belts (third and fourth) and normalized hopper masses with dotted shown bounds (bottom) for a prediction horizon of 160 steps.
As required, the machine hopper mass reaches the start level at the end of the prediction horizon. In comparison, the actual control only aims at filling the machine hopper to the maximum. Therefore, the machine hopper is usually full. If the moulding sand demand is less than the maximum capacity of the mixers, the mixer hoppers are filled to the maximum as well. Because of the batch process with fixed masses of approximately $50 \%$ of the mixer hopper capacity, the mixer hopper masses fluctuate in range of approximately 40-100\%. The variation of the disturbance has a remarkable impact on the optimization potential of energy saving. The lower and the more precisely the moulding sand demand is known, the greater the potential.

\section{B. Production Plant Test}

Initial results of the previous optimal control approach at the production plant of the exemplified foundry show a reduction for energy consumption. The optimal control problem from section III is solved at each time step $\Delta t$ in real-time with configuration from table II. The real-time test is performed for 120 time-steps (30 minutes) with an prediction horizon of 160 steps on a typical day with low moulding sand demand, see Fig. 3. The mixers switch-off four times, see Fig. 3 (top, sector A). The hopper masses are scaled to their maximum and vary in range between the minimum and the maximum bound, see Fig. 3, (bottom). The reduction in energy consumption is approximately $7.9 \%$.
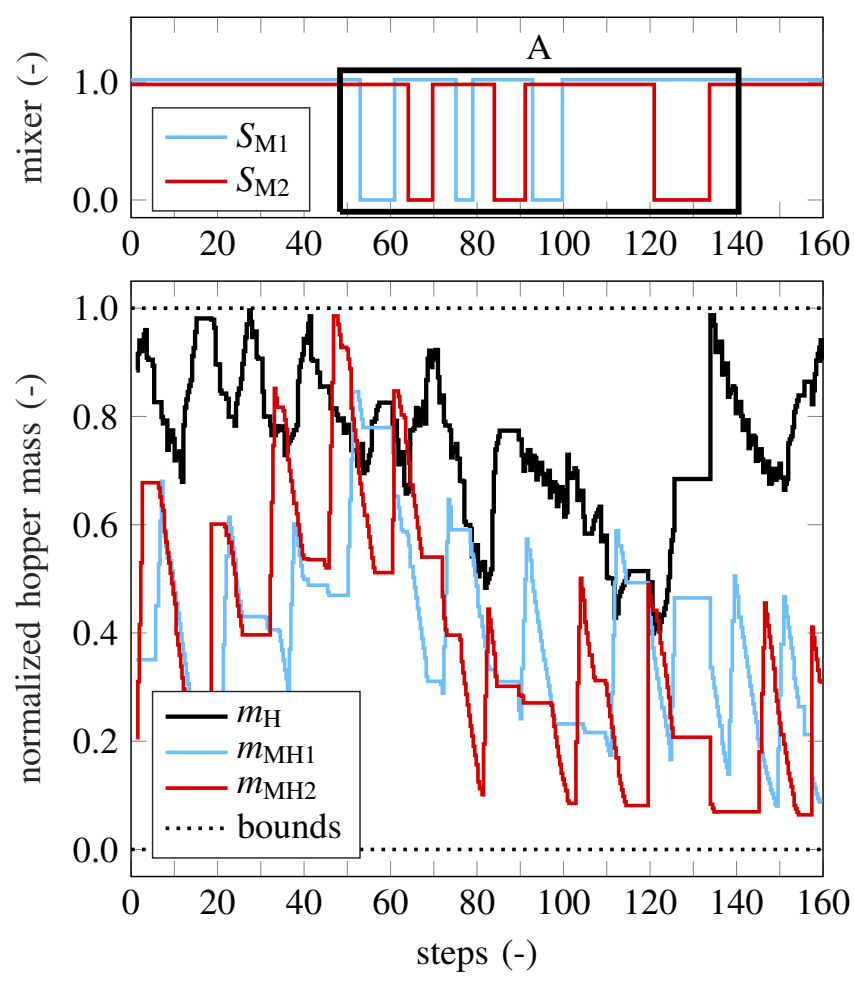

Fig. 3. Initial test of the optimal control approach in a model predictive control scheme on the production plant. Time series from 13.11.2020 with the mixer states (top) and the measured hopper masses (bottom) for a prediction horizon of 160 steps. 


\section{CONCLUSION}

This paper illustrates an optimal control approach for the energy consumption of interdependent mixing machines in foundry. Due to the constant mass per batch, the dosing and the mixing process can be modelled as dead-time systems with normalized masses and binary state variables. The mass on the transport belt is described as a discrete dead-time system and the hopper masses are defined as discrete linear systems. The switching function for the mixer state contains integer values. Applying constraint pushing separates the starts of the mixers and keeps the problem formulation linear. The economic cost function can be formulated linearly, among other reasons due to the modelling of the mixer idle time from the state information of the normalized masses. A soft constraint formulation for the bounds of the hopper masses improves the solvability by increasing the number of possible solutions near the boundaries.

The solution of the optimal control problem suggests savings in energy consumption in comparison to the actual heuristic control. The production plant test shows the capability of the presented approach. It yields a remarkable reduction of energy consumption by scheduling the batches and switch-off the mixers instead of idle times. Thus, the presented method contributes to the qualification of optimal control for foundry applications.

Future work will focus on the transfer of the optimal control problem into model predictive control scheme and its application at the production plant. Furthermore, the disturbance is essential for the optimization. Actually the disturbance is not predicted. In future studies, the energy consumption may be further decreased by adaptive moulding sand demand prediction, e.g. based on neural networks. Furthermore, improving the interaction of model and solver is possible. Investigations of the solver performance show a high computational effort when detecting symmetries and preforming restarts in the optimization process. Adjusting the model to the solver or optimizing solver settings itself can lead to further improvements. Subsequently, an endurance test to evaluate the presented method under a variety of environmental influences is planned at the foundry.

\section{ACKNOWLEDGMENT}

We would like to thank the companies Heinrich Meier Eisengießerei GmbH \& Co. KG, Rahden and Künkel Wagner Germany $\mathrm{GmbH}$, Alfeld for their generous support of this research work.

\section{REFERENCES}

[1] W. Tilch, H. Polzin, and M. Franke, Praxishandbuch bentonitgebundener Formstoff, 1st ed. Fachverlag Schiele \& Schön, 2015.

[2] A. Rose, A. Seel, B. Luck, and M. Grotjahn, "Lstm water prediction for feedforward control of moulding sand compressibility," 21th IFAC World Congress, 2020.

[3] D. M. Trinowski, "Die Gießerei-Industrie in Europa und den USA - ein Vergleich," Giesserei, vol. 103, no. 8, pp. 58-63, 2016.

[4] A. Sauer and T. Bauernhansl, Energieeffizienz in Deutschland - eine Metastudie, 2nd ed. Springer Vieweg, 2016.
[5] M. Blesl and A. Kessler, Energieeffizienz in der Industrie, 2nd ed. Springer Vieweg, 2017.

[6] W. Ernst, "Energieeinsparpotential in der Sandaufbereitung," Giesserei, vol. 107 , no. 10 , pp. 50-56, 2020.

[7] R. F. Teixeiri Jr., F. C. Fernandes, and P. N. A., "Binary integer programming formulations for scheduling in market-driven foundries," Computer \& Industrial Enginerring, vol. 59, no. 10, pp. 425-425, 2010. [Online]. Available: http://www.sciencedirect.com/science/article/pii/S0360835210001427

[8] M. Kong, J. Pei, J. Xu, X. Liu, X. Yu, and P. M. Pardalos, "A robust optimization approach for integrated steel production and batch delivery scheduling with uncertain rolling times and deterioration effect," International Journal of Production Research, vol. 58, no. 17, pp. 5132-5154, 2020. [Online]. Available: https://doi.org/10.1080/00207543.2019.1693659

[9] X. Zhang, G. Hug, Z. Kolter, and I. Harjunkoski, "Computational approaches for efficient scheduling of steel plants as demand response resource," 2016 Power Systems Computation Conference (PSCC), pp. 1-7, June 2016.

[10] S. Yu and Y. Hung, "Comparisons of three mixed integer programming models for parallel machine scheduling," 2016 IEEE International Conference on Industrial Engineering and Engineering Management, pp. 917-921, 2016.

[11] P. Kongsri and J. Buddhakulsomsiri, "A mixed integer programming model for unrelated parallel machine scheduling problem with sequence dependent setup time to minimize makespan and total tardiness," 2020 IEEE 7th International Conference on Industrial Engineering and Applications (ICIEA), pp. 605-609, April 2020.

[12] N. Frigerio and A. Matta, "Analysis on energy efficient switching of machine tool with stochastic arrivals and buffer information," IEEE Transactions on Automation Science and Engineering, vol. 13, no. 1, pp. 238-246, Jan 2016.

[13] S. I. Gass and M. C. Fu, Encyclopedia of Operations Research and Management Science. Springer US, 2013.

[14] J. Lofberg, "Yalmip : a toolbox for modeling and optimization in matlab," in 2004 IEEE International Conference on Robotics and Automation (IEEE Cat. No.04CH37508), Sep. 2004, pp. 284-289. 